\title{
Anti-synthetase syndrome: Validity of ANA as a screening tool - The Oxford interstitial lung disease service experience
}

\author{
Thomas Nicholson ${ }^{1}$, Andrew Woods ${ }^{2}$, Joel David ${ }^{3}$ and Rachel Hoyles ${ }^{1 *}$ \\ ${ }^{1}$ Oxford Centre for Respiratory Medicine, Churchill Hospital, Oxford, OX3 7LE, UK \\ ${ }^{2}$ Clinical Immunology Department, Churchill Hospital, Oxford, OX3 7LE, UK \\ ${ }^{3}$ Department of rheumatology, Nuffield Orthopaedic Centre, Oxford, OX3 7HE, UK
}

Anti-synthetase Syndrome (ASS) is characterized by Interstitial Lung Disease (ILD), myositis, arthropathy, fever, Raynaud's, and mechanics hands associated with anti-synthetase antibodies (including Jo-1, PL-7 and PL-12). ILD is the major determinant of mortality in ASS [1]. Anti-nuclear Antibody testing (ANA) is commonly used to screen for autoimmune diseases. If negative, in many centres further immunology testing with Extractable Nuclear Antigens (ENA) is not performed. We report a retrospective review of our centre's cohort of patients with ASS-ILD that highlights the inadequacy of this approach.

We retrospectively examined consecutive patients in the Oxford ILD and Rheumatology services with ASS-ILD between 2009-2014. CT scans were reviewed to identify the pattern of ILD. Immunology, lung function and medication were identified from patient records.

Twenty-four patients with ASS-ILD were identified over the 5-year period. They were aged 33-78 years (mean 54 years). 9 were male and 15 female. Disease severity was assessed by lung function at presentation: FVC $42-118 \%$ (mean $77.9 \%$ ) predicted, TLco 10-99\% (mean 56\%) predicted.

Only 1 out of 24 was ANA positive ( $\geq 1 / 80$ dilution). 18 of 24 (75\%) had a positive ENA screen (ELISA): 13 Jo-1; 4 Jo-1 \& Ro-52; 1 Ro. 6 (25\%) patients had a negative ENA screen. 5 patients with negative ENA ELISA had a positive myositis blot: 1 Jo-1; 3 PL-7; 1 PL-12, and

Copyright: (C2016 Nicholson T. This is an open-access article distributed under the terms of the Creative Commons Attribution License, which permits unrestricted use, distribution, and reproduction in any medium, provided the original author and source are credited.
1 was negative for all 3 autoantibodies. Of 7 patients who were Jo- 1 positive on ENA ELISA, 4 had a negative Jo-1 myositis blot.

We examined the CT patterns of disease: organizing pneumonia (OP; $n=7)$, non-specific interstitial pneumonia (NSIP; n=7), OP/NSIP overlap $(n=9)$, acute interstitial pneumonia (AIP; $n=1$ ). There was no relationship between anti-synthetase antibody and CT pattern.

The identification of an ASS antibody significantly changed management in most patients; 17 were treated with (IV) cyclophosphamide and rituximab was added to 8 cases.

The identification of an anti-synthetase antibody is central to diagnosis and significantly impacts on patient management. This data demonstrates that ANA is an inadequate screening test for ASS, as employed in several institutions. If ASS is clinically suspected, ENA testing for cytoplasmic tRNA-synthetase enzymes should be performed despite a negative ANA result, as ANA is a subjective nuclear rather than cytoplasmic stain, with poor sensitivity for anti-synthetase antibodies. The data also demonstrate that either an ENA ELISA or a myositis blot used in isolation lack the required sensitivity. These tests need to be used in combination to avoid false negative results.

\section{References}

1. Saketkoo LA, Ascherman DP, Cottin V, Stine LS, Danoff SK, et al. (2010) Interstitial Lung Disease in Idiopathic Inflammatory Myopathy. Curr Rheumatol Rev. 6: 108-119. [Crossref]
Correspondence to: Rachel Hoyles, Oxford Interstitial Lung Disease Service Lead, Oxford University Hospitals NHS Foundation Trust, Oxford Centre for Respiratory Medicine, Churchill Hospital, Old Road, Oxford OX3 7LE,UK, Tel: 01865 225223; E-mail: Rachel.Hoyles@ouh.nhs.uk

Received: July 29, 2016; Accepted: August 15, 2016; Published: August 18, 2016 